\title{
CURVATURE ESTIMATES FOR MINIMAL SURFACES
}

\author{
SOOK HEUI JUN
}

(Communicated by Clifford J. Earle, Jr.)

\begin{abstract}
In this article, we estimate the Gaussian curvature of nonparametric minimal surfaces by using the properties of univalent harmonic mappings.
\end{abstract}

\section{INTRODUCTION}

Recently, Hengartner and Schober [3] studied the class $\Sigma_{H}$ of all complexvalued, harmonic, orientation-preserving, univalent mappings $f$ defined on $\Delta=\{z:|z|>1\}$ that are normalized at infinity by $f(\infty)=\infty$. Such functions admit the representation

$$
f(z)=h(z)+\overline{g(z)}+A \log |z|,
$$

where

$$
h(z)=\alpha z+\sum_{k=0}^{\infty} a_{k} z^{-k} \quad \text { and } \quad g(z)=\beta z+\sum_{k=1}^{\infty} b_{k} z^{-k}
$$

are analytic in $\Delta$ and $0 \leq|\beta|<|\alpha|$. In addition, $\tilde{a}=\overline{f_{z}} / f_{z}$ is analytic and satisfies $|\tilde{a}(z)|<1$.

Now let $\Omega$ be a doubly connected domain in the extended $w$-plane having the point $w=\infty$ as one of its boundary continua. Let $S$ be a nonparametric surface over $\Omega$ given by

$$
S=\{(u, v, \varphi(u, v)): u+i v \in \Omega\} .
$$

We shall associate with every solution $\varphi(u, v)$ of the classical equation of minimal surfaces,

$$
\left(1+\varphi_{v}^{2}\right) \varphi_{u u}-2 \varphi_{u} \varphi_{v} \varphi_{u v}+\left(1+\varphi_{u}^{2}\right) \varphi_{v v}=0,
$$

the functions

$$
\begin{gathered}
\psi(u, v)=\int \frac{\varphi_{u} d v-\varphi_{v} d u}{\sqrt{1+\varphi_{u}^{2}+\varphi_{v}^{2}}} ; \\
F=\varphi+i \psi \\
\omega=\frac{\varphi_{u}-i \varphi_{v}}{1+\sqrt{1+\varphi_{u}^{2}+\varphi_{v}^{2}}} .
\end{gathered}
$$

Received by the editors June 29, 1990.

1980 Mathematics Subject Classification (1985 Revision). Primary 53A10, 30C50, 30C45. 
Note that the integral defining $\psi$ is path-independent by virtue of (1.2) and that $\psi$ is determined but for an additive constant. Also, we have $|\omega|<1$. The functions $F$ and $\omega$ are to be considered as defined on $S$. While $\omega$ is single-valued on this surface, $F$ may be multi-valued, but its branches differ only by constants.

If $\varphi(u, v)$ is interpreted as the potential of a flow of a hypothetical "Chaplygin gas" whose density $\rho$ and speed $q$ are connected by the relation

$$
\rho^{2}\left(1+q^{2}\right)=1
$$

then $\psi$ is the stream-function, $F$ the complex potential, and $\varphi_{u}-i \varphi_{v}$ the conjugate complex velocity (cf. [1]).

From now on, assume that $\varphi$ is nonconstant; that is, $S$ is not a horizontal plane. Then $S$ is a minimal surface if and only if $S$ admits a conformal reparametrization of the form

$$
S=\left\{\left(\operatorname{Re} G_{1}(z), \operatorname{Re} G_{2}(z), \operatorname{Re} F(z)\right): z=x+i y \in \Delta\right\},
$$

where

$$
G_{1}(z)=\frac{1}{2} \int F^{\prime}\left(\frac{1}{\omega}-\omega\right) d z, \quad G_{2}(z)=-\frac{i}{2} \int F^{\prime}\left(\frac{1}{\omega}+\omega\right) d z .
$$

The function

$$
f(z)=\operatorname{Re} G_{1}(z)+i \operatorname{Re} G_{2}(z)=\frac{1}{2} \int \frac{F^{\prime}(z)}{\omega(z)} d z-\frac{1}{2} \overline{\int \omega(z) F^{\prime}(z) d z}
$$

is a univalent harmonic mapping from $\Delta$ onto $\Omega$ with $f(\infty)=\infty$ and $\overline{f_{\bar{z}}} / f_{z}=$ $-\omega^{2}$. In $\Delta$, the variables $F$ and $\omega$ considered as functions of $z$ are regular analytic, $d F / d z$ and $\omega$ are single-valued, and $(1 / \omega)(d F / d z) \neq 0, \infty$. The function $\omega(z)$ is regular at $z=\infty$ and $|\omega(\infty)|<1$. Furthermore, $F^{\prime}(z) / \omega(z)$ is regular and different from zero at $\infty$. Also observe that we may assume $f$ is orientation-preserving and that we may obtain any other set of isothermal parameters by applying a conformal mapping to $\Delta$ (cf. [1]).

Note that $\omega(u, v)=0$ if and only if the unit normal vector to the surface $\mathbb{N}=\left(-\varphi_{u},-\varphi_{v}, 1\right) / \sqrt{1+\varphi_{u}^{2}+\varphi_{v}^{2}}$ is $(0,0,1)$ at $(u, v, \varphi(u, v))$. Since $f(z) \in \Sigma_{H}, f$ has the representation (1.1).

In this article, we estimate the Gaussian curvature of nonparametric minimal surfaces over $\Omega$ by using the properties of univalent harmonic mappings $f$ in $\Delta$ with $f(\infty)=\infty$. Our estimates are stated as inequalities (2.6) and (2.7) in Theorem 2.4 .

\section{Curvature estimates}

We want to discuss the Gaussian curvature $K$ for nonparametric minimal surfaces $S$. Since $G_{1}^{\prime}(z)^{2}+G_{2}^{\prime}(z)^{2}+F^{\prime}(z)^{2}=0$ by virtue of $(1.3)$, the Gaussian curvature at each point is given by

$$
K=-\left[\frac{4\left|\mathscr{G}^{\prime}\right|}{|\mathscr{F}|\left(1+\mid \mathscr{G}^{2}\right)^{2}}\right]^{2},
$$

where $\mathscr{F}=G_{1}^{\prime}-i G_{2}^{\prime}$ and $\mathscr{G}=F^{\prime} / \mathscr{F}$, by Lemma 9.1 in [4]. Since $\mathscr{F}=-F^{\prime} \omega$ and $\mathscr{G}=-1 / \omega$, we have

$$
|K|=\frac{16}{\left|F^{\prime} / \omega\right|^{2}} \frac{\left|\omega^{\prime}\right|^{2}}{\left(1+|\omega|^{2}\right)^{4}} .
$$


From (1.4) and (1.1), we have that

$$
2\left(h^{\prime}-g^{\prime}+(A-\bar{A}) /(2 z)\right) /\left(1+\omega^{2}\right)=F^{\prime} / \omega .
$$

Hence,

$$
|K|=\frac{4\left|\omega^{\prime}\right|^{2}\left|1+\omega^{2}\right|^{2}}{\left|h^{\prime}-g^{\prime}+(A-\bar{A}) /(2 z)\right|^{2}\left(1+|\omega|^{2}\right)^{4}} .
$$

Furthermore, the estimate $\left|\omega^{\prime}\right| /\left(1-|\omega|^{2}\right) \leq 1 /\left(|z|^{2}-1\right)$ from Schwarz's lemma for $|z|>1$ implies

$$
|K| \leq \frac{4 T(z)}{\left|h^{\prime}-g^{\prime}+(A-\bar{A}) /(2 z)\right|^{2}\left(|z|^{2}-1\right)^{2}},
$$

where $T(z)=\left(1-|\omega|^{2}\right)^{2}\left|1+\omega^{2}\right|^{2} /\left(1+|\omega|^{2}\right)^{4}$.

Now we restrict our attention to the case of $\Omega=\mathbb{C} \backslash[a, b]$, where $[a, b]$ is a real line segment in the complex plane.

For $f=u+i v$, we necessarily have $v=0$ on $|z|=1$, and $f(\infty)=\infty$ gives

$$
v(z)=\operatorname{Im}\left\{\alpha z+a_{0}+\overline{\beta z}\right\}+\tilde{r}(z)+(\operatorname{Im} A) \log |z|,
$$

where $\tilde{r}$ is harmonic and vanishes at infinity (cf. [3]). Let $R(\zeta)=\tilde{r}(1 / \zeta)$; then $R(\zeta)$ is harmonic on $|\zeta|<1$ and $R(0)=0$. So we have

$$
0=R(0)=\frac{1}{2 \pi} \int_{0}^{2 \pi} R\left(e^{i \phi}\right) d \phi=\frac{1}{2 \pi} \int_{0}^{2 \pi} \operatorname{Im}\left\{\bar{\alpha} e^{i \phi}+\bar{a}_{0}+\beta e^{-i \phi}\right\} d \phi,
$$

since $v=0$ on $|z|=1$ and $-\operatorname{Im}\left\{\alpha e^{-i \phi}+a_{0}+\bar{\beta} e^{i \phi}\right\}=\operatorname{Im}\left\{\bar{\alpha} e^{i \phi}+\bar{a}_{0}+\beta e^{-i \phi}\right\}$. This implies $\operatorname{Im} \bar{a}_{0}=0$. Thus $a_{0}$ is real. Therefore, $v(z)=\operatorname{Im}\{\alpha z+\overline{\beta z}\}+$ $\tilde{r}(z)+(\operatorname{Im} A) \log |z|$. Solving the Dirichlet problem for $\tilde{r}$, we conclude that $\tilde{r}(z)=\operatorname{Im}\{(\bar{\alpha} / z)+(\beta / \bar{z})\}$, and therefore

$$
v(z)=\operatorname{Im}\{\alpha z+\overline{\beta z}+(\bar{\alpha} / z)+(\beta / \bar{z})\}+(\operatorname{Im} A) \log |z| .
$$

In the representation (1.1), it follows that

$$
\operatorname{Im}\{h(z)+\overline{g(z)}\}=\operatorname{Im}\{\alpha z+\overline{\beta z}+(\bar{\alpha} / z)+(\beta / \bar{z})\} .
$$

Taking derivatives with respect to $z$, we obtain

$$
h^{\prime}-g^{\prime}=(\alpha-\beta)-(\overline{\alpha-\beta}) z^{-2} \text {. }
$$

We can easily show that we are free to normalize $\alpha-\beta$ to be real and positive. In that case,

$$
h^{\prime}-g^{\prime}=(\alpha-\beta)\left(1-z^{-2}\right) .
$$

This implies $h-g=(\alpha-\beta)\left(z+z^{-1}\right)+$ constant. But $h-g=(\alpha-\beta) z+$ $a_{0}+\left(a_{1}-b_{1}\right) z^{-1}+\sum_{k=2}^{\infty}\left(a_{k}-b_{k}\right) z^{-k}$. By comparing these two equations, we get $a_{1}-b_{1}=\alpha-\beta$ and $a_{k}=b_{k}$ for $k \geq 2$. Thus we have $h-g=$ $(\alpha-\beta)\left(z+z^{-1}\right)+a_{0}$.

Now we want to find, at a fixed point of $\Omega$, a bound for $|K|$ for the family of nonparametric minimal surfaces that lie over $\Omega=\mathbb{C} \backslash[a, b]$.

Let $p=p_{1}+i p_{2} \in \Omega$; then there exists $z=r e^{i \theta}$ such that $f(z)=p$. From (2.1) we get

$$
p_{2}=(\alpha-\beta)\left(r-r^{-1}\right) \sin \theta+(\operatorname{Im} A) \log r .
$$


Equation (2.2) implies

$$
\begin{aligned}
\left|h^{\prime}-g^{\prime}+(A-\bar{A})(2 z)^{-1}\right|^{2}= & r^{-2}\left(\operatorname{Im} A+(\alpha-\beta)\left(r^{2}+1\right) r^{-1} \sin \theta\right)^{2} \\
& +(\alpha-\beta)^{2}\left(1-r^{-2}\right)^{2} \cos ^{2} \theta
\end{aligned}
$$

So,

$$
|K| \leq \frac{4}{\left(r-r^{-1}\right)^{2}\left(\operatorname{Im} A+(\alpha-\beta)\left(r+r^{-1}\right) \sin \theta\right)^{2}+\left(r-r^{-1}\right)^{4}(\alpha-\beta)^{2} \cos ^{2} \theta},
$$

since $T\left(r e^{i \theta}\right) \leq 1$.

If $A$ is real, then $p_{2}=(\alpha-\beta)\left(r-r^{-1}\right) \sin \theta$. In this case,

$$
|K| \leq \frac{4}{\left(r-r^{-1}\right)^{4}(\alpha-\beta)^{2}+4 p_{2}^{2}} .
$$

And the necessary and sufficient condition for $p_{2}=0$ is $\sin \theta=0$, since $\alpha-\beta$ and $r-r^{-1}$ are positive real for $r>1$. So we obtain the estimates

$$
|K| \leq \frac{4}{p_{2}^{2}\left(4+p_{2}^{2}(\alpha-\beta)^{-2} \sin ^{-4} \theta\right)} \quad \text { if } p_{2} \neq 0
$$

and

$$
|K| \leq \frac{4}{(\alpha-\beta)^{2}\left(r-r^{-1}\right)^{4}} \quad \text { if } p_{2}=0 .
$$

But the parameters $r$ and $\theta$ in these estimates are not geometric quantities. So we want to replace these by estimates of a more geometric nature, for example, in terms of $p$.

The following result is due to Hengartner and Schober [3].

Lemma 2.1 [3, Theorem 4.3]. Let $\Sigma_{H R}=\left\{f \in \Sigma_{H}: \alpha=1, a_{0}=\beta=0\right.$, $\mathbb{C} \backslash f(\Delta) \subset \mathbb{R}\}$. Then the diameter $D_{f}$ of $\mathbb{C} \backslash f(\Delta)$ satisfies $\max _{\Sigma_{H R}} D_{f}=2 \pi$. Equality occurs if and only if $f(z)=z-\bar{z}^{-1}+2 \arg \left(\frac{1+i / z}{1-i / z}\right)$.

In order to obtain estimates of a more geometric nature, the following lemmas will be useful.

Lemma 2.2. If $f \in \Sigma_{H}$ and $f$ extends to be of bounded variation on $|z|=1$, then

$$
\mid \begin{aligned}
& \alpha+\bar{b}_{1}|\leq L /(2 \pi), \quad| b_{n} \mid \leq L /(2 n \pi) \quad \text { for } n \geq 2, \\
& \beta+\bar{a}_{1} \mid \leq L /(2 \pi), \quad \text { and }\left|a_{n}\right| \leq L /(2 n \pi) \quad \text { for } n \geq 2,
\end{aligned}
$$

where $L$ is the length of $f(|z|=1)$.

Proof. By integration by parts, we have

$$
\left|\int_{|z|=1} z^{n} d f\right|= \begin{cases}2 \pi\left|\alpha+\bar{b}_{1}\right| & \text { if } n=-1 \\ 2 \pi\left|-n \bar{b}_{-n}\right| & \text { if } n \leq-2 \\ 2 \pi\left|a_{1}+\bar{\beta}\right| & \text { if } n=1 \\ 2 \pi n\left|a_{n}\right| & \text { if } n \geq 2\end{cases}
$$

Since $\left|\int_{|z|=1} z^{n} d f\right| \leq \int_{|z|=1}|d f|=L$, we have

$$
\left|\alpha+\bar{b}_{1}\right| \leq L /(2 \pi), \quad\left|a_{1}+\bar{\beta}\right| \leq L /(2 \pi),
$$

and

$$
n\left|b_{n}\right| \leq L /(2 \pi), \quad n\left|a_{n}\right| \leq L /(2 \pi) \quad \text { for } n \geq 2
$$


These inequalities are equivalent to the desired ones. Q.E.D.

Lemma 2.3. If $\omega(\infty)=0$, then $\beta=A=0$ and

$$
\frac{b-a}{2 \pi} \leq \alpha \leq \frac{b-a}{\pi} .
$$

Proof. Since $\overline{f_{\bar{z}}} / f_{z}=-\omega^{2}$, it follows that $\overline{f_{\bar{z}}}(\infty)=\beta=0 . \tilde{f}(z)=(f(z)-$ $\left.a_{0}\right) / \alpha \in \Sigma_{H R}$ and $\mathbb{C} \backslash \tilde{f}(\Delta)=\left[\left(a-a_{0}\right) / \alpha,\left(b-a_{0}\right) / \alpha\right]$ imply that $(b-a) / \alpha \leq$ $2 \pi$ by Lemma 2.1. So we have $(b-a) / 2 \pi \leq \alpha$. We can easily show that $\lim _{r \rightarrow 1^{+}} f\left(r e^{i \theta}\right)$ exits a.e. and belongs to $[a, b]$. Also, $f$ is orientationpreserving near $\partial \Delta$. So $f$ extends to be of bounded variation on $|z|=1$. Now apply Lemma 2.2; then we obtain

$$
\left|\alpha+\bar{b}_{1}\right| \leq(b-a) / \pi \text {. }
$$

In order to obtain an upper bound for $\alpha$, we need to know the coefficients of $f$ in terms of the coefficients of $\omega$ and $F^{\prime}$. Since $\omega(\infty)=0$ and $F^{\prime} / \omega \neq$ $0, \infty$, we have series expansions

$$
\omega(z)=\sum_{\nu=n}^{\infty} x_{\nu} z^{-\nu} \quad \text { and } \quad F^{\prime}(z)=\sum_{\nu=n}^{\infty} y_{\nu} z^{-\nu},
$$

where $x_{n} y_{n} \neq 0$ and $n \geq 1$. Substitute these two series into (1.4) and obtain

$$
\begin{aligned}
f(z) & =\frac{\frac{1}{2}\left(y_{n} x_{n}^{-1} z+\left(y_{n+1} x_{n}^{-1}-y_{n} x_{n+1} x_{n}^{-2}\right) \log z+\cdots\right)}{-\frac{1}{2}\left((1-2 n)^{-1} x_{n} y_{n} z^{1-2 n}-(2 n)^{-1}\left(y_{n} x_{n+1}+x_{n} y_{n+1}\right) z^{-2 n}+\cdots\right)}+\text { constant. }
\end{aligned}
$$

Since $f(z)$ must be a single-valued function, we must have that

$$
y_{n+1} x_{n}^{-1}-y_{n} x_{n+1} x_{n}^{-2}=0 \text {. }
$$

So we do not have a logarithmic term in $f(z)$, i.e., $A=0$, and

$$
\alpha=y_{n} /\left(2 x_{n}\right), \quad b_{1}= \begin{cases}\left(x_{1} y_{1}\right) / 2 & \text { if } n=1, \\ 0 & \text { if } n>1 .\end{cases}
$$

Note that $y_{1}$ must be real because $\varphi=\operatorname{Re} F(z)$ and $\varphi$ is single-valued. Thus, we have

$$
\left|\alpha+\bar{b}_{1}\right|= \begin{cases}\alpha\left(1+\left|x_{1}\right|^{2}\right) & \text { if } n=1 \\ \alpha & \text { if } n>1 .\end{cases}
$$

From (2.5), we obtain $\alpha\left(1+\left|x_{1}\right|^{2}\right) \leq(b-a) / \pi$. This implies that $\alpha \leq$ $(b-a) / \pi$. Q.E.D.

Remark. $\omega(\infty)=0$ if and only if the unit normal vector to the surface with the standard orientation at $\infty$

$$
\mathbb{N}_{\infty}=\left(\frac{2 \operatorname{Re}\{-1 / \omega\}}{1+|\omega|^{-2}}, \frac{2 \operatorname{Im}\{-1 / \omega\}}{1+|\omega|^{-2}}, \frac{1-|\omega|^{2}}{1+|\omega|^{2}}\right)
$$

is $(0,0,1)$. 
Theorem 2.4. If $S$ is a nonparametric minimal surface over the slit plane $\Omega=$ $\mathbb{C} \backslash[a, b]$ and if the unit normal to the surface at $\infty$ is $(0,0,1)$, then we have

$$
\begin{gathered}
|K(p)| \leq \frac{4(b-a)^{2}}{p_{2}^{2}\left[\pi^{2} p_{2}^{2}+4(b-a)^{2}\right]} \quad \text { if } p_{2} \neq 0, \\
|K(p)| \leq \frac{4(b-a)^{2}}{\pi^{2} d^{4}} \quad \text { if } p_{2}=0,
\end{gathered}
$$

where $p=p_{1}+i p_{2} \in \Omega$ and $d$ is the distance from $p$ to $[a, b]$.

Proof. Let $f\left(r e^{i \theta}\right)=p$. If $p_{2} \neq 0$, then we have (2.3). By applying Lemma 2.3, we obtain

$$
|K(p)| \leq \frac{4(b-a)^{2}}{p_{2}^{2}\left[\pi^{2} p_{2}^{2}+4(b-a)^{2}\right]} .
$$

If $p_{2}=0$, then we have $\sin \theta=0$, and so $f(s)=p$ for some real $s$. From $\beta=A=0$, we have $f(z)=\alpha z+a_{0}+\left(b_{1}+\alpha\right) z^{-1}+\bar{b}_{1} \bar{z}^{-1}+2 \operatorname{Re} \sum_{k=2}^{\infty} a_{k} z^{-k}$. If $z$ is real, then $f(z)$ is real because $a_{0}$ is real. Since $\alpha$ is positive real, we have $f(r)>b$ for sufficiently large $r>1$. This implies that $\lim _{r \rightarrow 1^{+}} f(r)=b$. From $\operatorname{Im}\{f\}=\operatorname{Im}\{h-g\}, \operatorname{Re}\{f\}=\operatorname{Re}\{h+g\}$, and $h^{\prime}+g^{\prime}=\frac{1-\omega^{2}}{1+\omega^{2}}\left(h^{\prime}-g^{\prime}\right)$, it follows that we have the representation

$$
\operatorname{Re} f(z)=\operatorname{Re}\left\{\alpha z+\int_{\infty}^{z}\left(\frac{1-\omega^{2}}{1+\omega^{2}}\left(h^{\prime}-g^{\prime}\right)-\alpha\right) d z\right\}+a_{0} .
$$

If $f(r)=p \quad(r>1)$, then $p>b$ and

$$
\begin{aligned}
p-b & =\operatorname{Re} \int_{1}^{r} \frac{1-\omega^{2}}{1+\omega^{2}}\left(h^{\prime}-g^{\prime}\right) d z \\
& =\operatorname{Re} \int_{1}^{r} \frac{1-\omega^{2}}{1+\omega^{2}} \alpha\left(1-z^{-2}\right) d z \quad(\text { from }(2.2)) \\
& =\alpha \int_{1}^{r} \operatorname{Re}\left\{\frac{1-\omega^{2}}{1+\omega^{2}}\right\}\left(1-x^{-2}\right) d x \\
& \leq \alpha \int_{1}^{r} \frac{x^{2}+1}{x^{2}-1}\left(1-x^{-2}\right) d x \quad(\text { from Schwarz's lemma for }|z|>1) \\
& =\alpha\left(r-r^{-1}\right) .
\end{aligned}
$$

Thus $p-b \leq \alpha\left(r-r^{-1}\right)$. From (2.4) and this, we have $|K| \leq 4 \alpha^{2} /(p-b)^{4}$. Apply Lemma 2.3; then we have $|K| \leq 4(b-a)^{2} /(p-b)^{4} \pi^{2}$ for $p>b$. Similarly, we obtain $|K| \leq 4(b-a)^{2} /(p-a)^{4} \pi^{2}$ for $p<a$. Q.E.D.

Unfortunately, the estimates of $|K(p)|$ are not sharp. In order to obtain estimates (2.6) and (2.7), inequalities $T(z) \leq 1$ and $\alpha \leq(b-a) / \pi$ are used. In the inequality $T(z) \leq 1$, equality for $z \neq \infty$ occurs if and only if $\omega(z)=$ $c / z(|c|=1)$. Assume that $\omega(z)=e^{i \gamma} / z$ for some $\gamma$; then $\left|\alpha+\bar{b}_{1}\right|=2 \alpha$ (cf. Proof of Lemma 2.3), and $\left|\alpha+\bar{b}_{1}\right| \leq(b-a) / \pi$ (cf. Lemma 2.2). Therefore, the strict inequality $\alpha<(b-a) / \pi$ holds in this case.

But the estimates (2.6) and (2.7) are good in the following sense. As $b-a \rightarrow \infty$, the estimate (2.6) reduces to the sharp estimate $|K(p)| \leq 1 / p_{2}^{2}$ from Hengartner and Schober [2]. For $b-a<\infty$, it is even smaller.

If $b=0$, then the estimate (2.7) is $4 a^{2} / \pi^{2} p^{4}$ when $p>0$. This bound is better than Hengartner and Schober's when $a^{2} / p^{2}<\pi^{2}(3+2 \sqrt{3}) / 48$. 


\section{REFERENCES}

1. Lipman Bers, Isolated singularities of minimal surfaces, Ann. Math. 53 (1951), 364-386.

2. W. Hengartner and G. Schober, Curvature estimates for some minimal surfaces, Complex Analysis, Articles Dedicated to Albert Pfluger on the Occasion of His 80th Birthday (J. Hersch and A. Huber, eds.), Birkhäuser, 1988, pp. 87-100.

3. __ Univalent harmonic functions, Trans. Amer. Math. Soc. 299 (1987), 1-31.

4. R. Osserman, A survey of minimal surfaces, Dover, 1986.

Mathematics and Statistics, St. Cloud State University, St. Cloud, Minnesota 56301 